\title{
Solid state effects in electron emission from atomic collisions near surfaces
}

\author{
Carlos O. Reinhold, Joachim Burgdörfer, Ronaldo Minniti, Stuart B. Elston \\ Physics Division, Oak Ridge National Laboratory, Oak Ridge, TN 37831-6373, USA, \\ and University of Tennessee, Knoxville TN 37996-1200, USA
}

We present a brief progress report of recent studies of the ejected electron spectra arising from glancing-angle ion-surface scattering involving collision energies of hundreds of $\mathrm{keV} / \mathrm{u}$. A broad range of electron energies and emission angles is analyzed containing prominent structures such as the convoy electron peak and the binary ridge. Particular emphasis is placed on the search for signatures of dynamic image interactions and multiple scattering near surfaces.

PACS: $79.20 . \mathrm{Nc}, 79.20 . \mathrm{Rf}, 34.50 . \mathrm{Fa}$

\section{DISCLAIMER}

This report was prepared as an account of work sponsored by an agency of the United States Government. Neither the United States Government nor any agency thereof, nor any of their employees, makes any warranty, express or implied, or assumes any legal liability or responsibility for the accuracy, completeness, or usefulness of any information, apparatus, product, or process disclosed, or represents that its use would not infringe privately owned rights. Reference herein to any specific commercial product, process, or service by trade name, trademark, manufacturer, or otherwise does not necessarily constitute or imply its endorsement, recommendation, or favoring by the United States Government or any agency thereof. The views and opinions of authors expressed herein do not necessarily state or reflect those of the United States Government or any agency thereof.

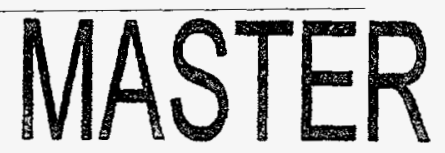

\author{
BISTABITION OF THIS DOCUMENT IS UNLMITED'p \\ "The submitted manluscript has been authored by \\ a contractor of the U.S. Government under \\ contract DE-ACO5-960R22464. Accordingly, the \\ U.S. Government retains a nonexclusive, royalty- \\ free license to publish or reproduce the published \\ form of this contribution, or allow others to do \\ so, for U.S. Government purposes."
}




\section{Introduction}

The study of kinetic electron emission in ion-solid collisions provides an important link between atomic physics and condensed matter physics. Comparative analysis of spectra from ion-atom, ion-solid (transmission) and ion-surface (glancing incidence) collisions affords the opportunity to extract detailed information about long-range image interactions and multiple scattering in the solid and near the surface.

The so-called convoy electron peak (CEP) represents an illuminating example for such differences. This peak was experimentally discovered two decades ago as a cusp-shaped structure in the spectra of electrons arising from ion-atom [1] and ion-solid (transmission) collisions [2] and ejected with velocities $\vec{v}_{e}$ close to the projectile velocity $\vec{v}_{p}$. Because cusp electrons recede from the target in close spatial correlation with the projectile, the behavior of the cross sections in the limit $\left|\vec{v}_{e}-\vec{v}_{p}\right| \rightarrow 0$ was found to be governed by threshold laws characteristic of the two-body final state interaction between the electron and the projectile.

Experiments concerning glancing-angle ion-surface interactions have revealed a prominent structure at forward ejection angles where the CEP is expected to appear in foil transmission experiments $[3,4,5,6,7,8,9,10,11]$. Compared to the CEP for transmission conditions, however, the structure for ion-surface collisions is dramatically broadened and shifted in energy. Despite these differences, the same term (i.e. "convoy") has been adopted to describe the peak for ion-surface scattering. The first evidence for a broad CEP was found by DeFerraris and Baragiola [3] for scattering of protons at an Al surface. A similar broadening was observed for semiconductor surfaces $[4,5]$. Concurrently, a shift of the CEP to electron velocities larger than $v_{p}$ was proposed [6] and independently measured [4] for projectile charges greater than one. Subsequently, large shifts of up to $100 \mathrm{eV}$ have been observed in several laboratories $[7,8,9,10,11,12]$.

A number of explanations of a shifted CEP have been proposed $[6,10,11,13,14,18]$ all of which agree that this effect is closely related to dynamic image interactions and appears for scattering at reasonably flat surfaces. At grazing angles of incidence the 


\section{DISCLAIMER}

Portions of this document may be illegible in electronic image products. Images are produced from the best available original document. 
impinging ion remains near the target surface for a very long time and, therefore, the effective final-state interaction in which the excited electrons evolve is quite different from ion-atom collisions and foil transmission experiments. The energy shift is identified as "convoy-electron acceleration" and the underlying picture is that of emitted electrons repelled to larger energies by the negative image of the projectile ion travelling in close proximity (see Figure 1).

The details of the microscopic mechanisms leading to the CEP are still under discussion. Clearly, emission of electrons near surfaces is a quite complex process and most theoretical descriptions have resorted to simplified models of the full collision problem. Very recently, we have undertaken the development of a comprehensive classical trajectory Monte Carlo (CTMC) description of fast electron emission [18, 19]. Our model is a combination of the CTMC method as applied to atomic collisions $[15,16]$ and the classical transport theory originally developed for transmission of ions or atoms through solids [17]. Accordingly, the initial close collisions between the projectile ion and electrons of the target and the subsequent transport through the surface region and out to asymptotic distances is treated on the same footing. This approach permits the non-pertubative treatment of the core potentials and long-range dynamic image potentials.

In this work we give a brief account of progress in this field as well as of open questions to be addressed in the future. Most of this work is based on [19] to which we defer the reader for technical details. Atomic units are used throughout.

\section{Dynamics of electron emission}

One fundamental assumption in the description of electron emission is the decoupling of the dynamics of the impinging ion from the electronic dynamics. First, an approximate ionic trajectory is determined. Subsequently, electrons are assumed to evolve according to a time-dependent Hamiltonian containing the trajectory $\vec{R}(t)$ of the projectile. The different microscopic interactions which govern the ionic and electronic dynamics are discussed below. 


\subsection{Ionic dynamics}

Consider the scattering of a heavy ion with ionic charge $Q_{p}$ and initial velocity $\vec{v}_{p}$ at a crystal surface. Microscopically, the impinging ion will undergo a series of repulsive collisions with the screened target nuclei at the crystal. If the incidence angle of the ion with respect to the surface is sufficiently small (a few milliradians) and the surface is sufficiently flat, the ion will be specularly reflected. More specifically, reflection occurs when the energy of the ion in the direction normal to the surface is smaller than the planar-averaged potential barrier provided by the topmost layer of nuclei (typically tens of $\mathrm{eV}$ ) (see e.g. [20] for a more detailed description).

A typical grazing ion trajectory is depicted in Fig. 2. The cloud of electrons in the crystal extends a few atomic units beyond the topmost layer up to a distance referred to as the "jellium edge". The ion trajectory is such that it penetrates this edge. In principle, the heavy ion will be slowed down by interactions with electrons. However, typical energy losses are less than $3 \%$ of the incident energy (e.g. [23]) which has a negligible effect in the evolution of fast electrons.

Note the disparity of path length scales in the directions parallel or perpendicular to the surface. The large path length of the ion along the surface implies that it interacts with many target atoms (which are only a few atomic units from each other). As a consequence, the ionic charge state of the ion should be expected to fluctuate in time due to (i) capture of electrons from the solid into bound states of the ion and (ii) ionization of electrons in bound states of the projectile caused by collisions with particles in the solid. Figure 2 shows that in a typical charge changing cycle for $0.3 \mathrm{MeV} / \mathrm{u} \mathrm{Li}^{+}{ }_{-} \mathrm{SnTe}$ collisions $Q_{p}$ changes about 12 times. Therefore, memory of the initial charge state is completely lost, not only when the final charge state is reached but already during the interaction process in the vicinity of the surface. The charge state fractions for a $0.3 \mathrm{MeV} / \mathrm{u} \mathrm{Li}^{+}$ion impinging on SnTe displays an abrupt decrease of the charge state fraction of $\mathrm{Li}^{+}$ions from a value of one to almost zero during the first $20 \%$ of the ion trajectory after crossing the jellium edge. Subsequently, a quasi charge state equilibration is achieved yielding 
charge state fractions of $\mathrm{Li}^{2+}$ and $\mathrm{Li}^{3+}$ of about $50 \%$ which persists until exit.

Direct experimental evidence of charge equilibration has been obtained in different laboratories, an example of which is shown in Figure 3 for $\mathrm{C}^{q+}-\mathrm{Si}$ collisions [21]. The exit charge state distribution of the projectile is, within the accuracy of the experiment, identical for all incident charge states. Moreover, indirect evidence exists that charge state equilibration is rapidly reached while the projectile is still in close proximity to the surface. By measuring the spectra of ejected electrons in coincidence with the final charge state of the emerging projectiles, Kimura et al [22] recently found evidence consistent with a very rapid charge state fluctuation and equilibration near the surface. Specifically, no correlation was found between the position of the convoy peak and the outgoing charge state of the ion.

The existence of the charge-changing cycle which the ion undergoes complicates the analysis of electron emission from surfaces. This is due to the fact that the spectra possess an intricate dependence on $Q_{p}$. Recently, we have found experimentally that, despite the charge state equilibration shown in Fig. 3, the shift of the CEP depends on the impinging charge state of the ion [12]. This implies that at the moment the dominant electron yield is produced, charge state equilibration may not yet have been achieved. At present, this result is not completely understood.

\subsection{Electronic dynamics}

Adopting an independent electron approach, electron-electron interactions can be accounted for through dynamic image interactions, static atomic screening, and collision kernels. Within this framework, a realistic effective Hamiltonian describing the time evolution of an electron with position vector $\vec{r}$ and momentum $\vec{p}$ is given by

$$
H_{e}(\vec{r}, \vec{p}, \vec{R}, t) \simeq H_{0}^{i}(\vec{r}, \vec{p}, t)+V(\vec{r}, t)-\vec{r} \cdot \vec{F}_{s t}(t)
$$

where $\mathrm{H}_{0}^{i}$ is the channel Hamiltonian which defines the unperturbed initial state of the electron and $V(\vec{r}, t)$ is a perturbation potential.

$F_{s t}$ in Eq. 1 is a stochastic force describing inelastic and elastic collisions in the vicinity 
of the surface. Elastic collisions represent elastic scattering of the electron at the screened heavy nuclei in the solid. Inelastic collisions consist of single-particle-single-hole and plasmon excitations of valence electrons. $F_{s t}(t)$ can be reduced to a stochastic sequence of momentum transfers $\Delta \vec{p}_{j}$ delivered to the electron at times $t_{j}$. The explicit determination of the sequence $\left(\Delta p_{j}, t_{j}\right)$ near the surface is complicated and can be found elsewhere [19]. Typically, the total mean free path of an electron near the surface is about 5-10a.u. and increases exponentially for increasing separations from the surface. Consequently, electrons emitted at small angles with respect to the surface should be expected to undergo a few collisions prior to escaping.

The electronic interaction potentials entering the channel Hamiltonian $H_{0}^{i}$ (Eq. 1) depends on the initial state. Electrons in the valence or conduction band can be treated as a free electron gas with a channel potential given by the surface barrier potential, $V_{b}$. Core electrons below the valence band are in well localized atomic-like orbitals and the core potential $V_{t}^{c}$ supporting these orbitals has the form of a muffin-tin potential derived from atomic core potentials.

The perturbation potential $V(\vec{r}, t)$ entering $\mathrm{H}_{e}$ is mainly given by $V(\vec{r}, t) \simeq V_{p}^{c}(\mid \vec{r}-$ $\vec{R}(t) \mid)+V_{p e}^{I}\left(\vec{r}, R_{z}(t)\right)$, where $V_{p}^{c}$ is the core potential of the impinging projectile and $V_{p e}^{I}$ is the image potential induced by the projectile. Assuming a linear response of the electrons in the crystal, the induced potential can be obtained as the solution of the Poisson equation for a moving particle near a "surface" which separates vacuum and a medium described by a dielectric response function. For ion velocities $v_{p} \gg v_{F}\left(v_{F}\right.$ being the Fermi velocity), the induced potential can be obtained using a frequency dependent classical dielectric function [25]. For lower velocities [11] a dispersive dielectric function must be utilized [24].

In Fig. 4 we display the total potential experienced by an electron initially in a target core state near a $\mathrm{Li}^{2+}$ ion moving parallel to a SnTe(001) surface. In addition to the core fields of the target $(\mathrm{T})$ and the projectile $(\mathrm{P})$, a wake pattern behind the ion and the surface barrier potential $V_{b}$ are also clearly visible. The wake is given by $V_{p e}^{I}$ which, 
near the surface, is quite different from the point-like image interactions inferred from Figure 1. The image "bump" behind the ion is related to an enhanced electron density in that region which effectively repels other electrons giving rise to the so-called "image acceleration".

\section{$3 \quad$ Electron emission spectra}

A cut of the CTMC emission spectrum in the $\left(v_{x}, v_{z}\right)$ scattering plane is displayed in Fig. 5 for $0.3 \mathrm{MeV} / \mathrm{u} \mathrm{Li}$ ions scattering from a $\mathrm{SnTe}$ surface. In order to clearly identify different structures, the total ejected electron spectrum has been decomposed into the components originating from valence band and from core levels. Both cuts exhibit the "binary ridge" [26], well-known from ion-atom collisions. This structure arises from quasi two-body collisions between a target electron and the impinging ion. Because of the large mass of the ion, conservation of energy and momentum implies that the final velocity of an electron initially at rest will be $v_{e}=\left|\vec{v}_{p}+\vec{v}_{p}^{\prime}\right|$, where $\vec{v}_{p}^{\prime}$ has arbitrary direction and magnitude $v_{p}^{\prime}=v_{p}$. This corresponds to a sphere centered at $v_{e}=v_{p}$ with a radius equal to $v_{p}$. The location of such a "binary sphere" agrees with the region of highest density for valence electrons but not for core electrons. The width of the sphere is due to the initial momentum distribution of electrons in the valence band. The absence of a comparable structure for core electrons is a direct consequence of their broad momentum distribution which is comparable to the projectile velocity.

The spectrum of core electrons is seen to peak in the forward direction $\left(v_{x}>>v_{y}, v_{z}\right)$ and for velocities $v_{e} \gtrsim v_{p}$. This region of high density corresponds to the so-called convoy electron peak (CEP) for ion-surface scattering which is caused by the attraction of the projectile field. The shift of the peak to the $v_{e}>v_{p}$ region is a direct consequence of the image potential induced by the impinging ion, $V_{p e}^{I}$. The microscopic mechanism leading to the peak can be viewed as rainbow scattering of electrons at the screened field of the ion, $V_{p e}^{I}[18]$. In order to illustrate the dramatic effects introduced by this potential we give in Fig. 6 a comparison of our full simulation with a simulation with $V_{p e}^{I}$ turned off. 
Inclusion of the image of the ion not only broadens and shifts the highest density region from $v_{e} \simeq v_{p}$ to $v_{e}>v_{p}$ but causes a pronounced void in the forward electron spectrum near the region $v_{e} \simeq v_{p}$. This depletion is a direct consequence of the expulsion of ejected electrons by the repulsive first half-wave of the induced wake potential in the immediate vicinity of the ion. It should be emphasized that this void is not due to losses by multiple scattering near the surface, which is included in both calculations of Fig. 6 .

The doubly differential absolute yield of ejected electrons as a function of the emission energy at various emission angles $\theta=\cos ^{-1}\left(v_{x} / v_{e}\right)$ (Fig. 7) is broken down into three distinct components: emission of valence electrons, direct emission of core electrons, emission of electrons transiently bound to the projectile but originating from target cores. The most striking observation is that valence electrons represent only a very small fraction of the total yield of fast electrons for the present collision system. Consequently, the sharp binary ridge structure for emission of valence electrons (Fig. 5) shows up only as a shoulder in the total yield of electrons. The most discernible structure of the total spectrum is the convoy electron peak at small emission angles which is primarily due to direct excitation of target core states. The dominance of core electron emission in the spectrum of fast electrons is in part due to the larger multiplicity by a factor $\sim 3.6$ of active core electrons in the $\mathrm{N}$-shell of $\mathrm{Sn}$ or Te compared to valence electrons. More importantly, the ion velocities considered in this work, $v_{p} \sim 3-4 a . u$., are close to the matching velocities of target core electrons in the N-shell of SnTe. Since direct electron capture from target states into low-lying continuum states of the projectile relies on the overlap of the momentum distributions of projectile and target states [27], core electrons in the $\mathrm{N}$ shell are strongly favored in complete analogy to electron capture in atom collisions. By contrast, direct ionization of valence electrons into states with velocities $\vec{v}_{e} \sim \vec{v}_{p}$ is very unlikely since the initial velocities of electrons in the valence band, which are smaller than the Fermi velocity $\left(v_{F} \sim 0.9 a . u .,\right)$, are small compared to $v_{p}$.

In other collision systems, emission of valence or conduction electrons may dominate, e.g. collisions at lower velocities [11] or experiments involving lighter target atoms such 
as pyrolytic graphite surfaces [28]. The latter target has only two core electrons per atom which are very tightly bound and possess orbital velocities of $\sim 6 a . u$.. Therefore, experiments involving ions with a few hundred $\mathrm{keV} / u$ energies will predominantly ionize valence electrons resulting in a pronounced binary peak.

While direct excitation of a valence electron into convoy states is quite unlikely, valence electrons may eventually end up in the region of the convoy peak as a consequence of electron transport, i.e. multiple scattering. Loosely speaking, this process is analogous to Thomas scattering in ion-atom collisions, in which electron capture of a quasi-free electron takes place by a double scattering sequence, first at the projectile and subsequently at the target. In the present case, the two scattering events are separated in time and space and take place on the energy shell. The second scattering center is provided by the array of target nuclei in the surface. The broad convoy peak originating from valence electrons (Fig. 7) results from this multiple scattering sequence. Multiple scattering is also important in other regions of the ejected electron spectrum, for example, for emission of energetic valence electrons at large angles. They originate from binary encounter electrons which are first emitted towards the inside of the solid and are subsequently scattered at target cores. At an emission angle of 60 degrees, this pathway to electron emission gives rise to a shoulder in the energy distribution of ejected valence electrons at an energy that is larger than that of the direct binary peak at that angle. Furthermore, multiple scattering is responsible for ionizing core electrons which are transiently captured to bound states of the projectile, contributing $10-20 \%$ of the convoy yield in this case.

In Figure 8 we compare the results of our simulation, summed over all contributions from the core and valence electrons with measurements for the triply differential yield of ejected electrons. Our simulation predicts a pronounced convoy electron peak which is considerably shifted to energies larger than the nominal convoy peak energy $E / E_{i}=$ 1. The peak position and width are in reasonable agreement with experiment but the calculated shift is slightly larger than the experimental one. 


\section{Concluding remarks}

Progress has been made in the study of electron emission from glancing-angle ion-surface scattering. We have learned that image interactions and multiple scattering play important roles in the emission process. Depending on the collision energy and the atomic structure of the crystal, the spectra is dominated by either valence or core electrons. In addition, the charge state of the impinging ion has been found to be time-dependent and, therefore, any study of the spectrum should include an analysis of the projectile charge during emission. For $v_{p} \gtrsim v_{F}$ the CEP shift has been found to be (i) a monotonically decreasing function of $v_{p}[10,12]$ and (ii) a monotonically increasing function of the plasma frequency of the solid, $w_{p},[8]$. This behavior is related to the fact that the projectile image force along the surface is proportional to $w_{p}^{2} / v_{p}^{2}$. For $v_{p} \lesssim v_{F}$ the opposite dependence on $v_{p}$ has been found which reflects the disappearance of the wake for slow ions [11].

Absolute yields of ejected electrons would provide a very sensitive and critical test of theoretical models. Unfortunately, no published data for absolute convoy electron yields are available. We have compared calculated yields with preliminary estimates of experimental yields kindly provided to us [29] and we have found that the calculated yields are about an order of magnitude larger than the measurements. At this moment, no convincing explanation can be put forward to reconcile this discrepancy. Experiments currently underway at ORNL using Si targets [12] may shed some light on this problem.

Theoretically, employing the hydrodynamical model [30] for the induced potential to treat non-linear effects in the regime of strong perturbation $Q_{p} / v_{p} \simeq 1$ appears an attractive pathway for improvement of the simulations. This may allow to extend the simulations to large projectile charges or down to collision velocities of the order or smaller than the Fermi velocities of the crystal [11]. A realistic quantum mechanical treatment for convoy electron emission for the present collision systems remains a major challenge.

One interesting point of controversy still persists: whether or not the CEP for impinging protons is shifted. Sanchez et al [11] have found a shifted CEP for proton projectiles seemingly in contradiction with previous observations $[4,5,10]$. Our simulations for pro- 
tons do predict a shifted CEP but they also appear to overestimate the shift for other ions as well.

Still, much work is needed to fully characterize solid state signatures and to reconcile theoretical calculations with experimental data.

Support for this work has been provided by the U.S. DOE, OBES, Div. of Chem. Sciences, under Contract No. DE-AC05-960R22464 with ORNL-LMER Corp. and by the NSF. 


\section{References}

[1] C. B. Crook and M. E. Rudd, Phys. Rev. Lett. 25, 1599 (1970).

[2] K. G. Harrison and M. W. Lucas, Phys. Lett. 33A, 142 (1986).

[3] L. F. de Ferraris and R. Baragiola, Phys. Rev. A 33, 4449 (1986).

[4] M. Hasegawa, K. Kimura, M. Mannami, J Phys. Soc. Jap. 57, 1834 (1988).

[5] H. Winter, P. Strohmeier, and J. Burgdörfer, Phys. Rev. A 39, 3895 (1989).

[6] J. Burgdörfer, Nuc. Inst. Meth. 24/25, 139 (1987).

[7] A. Koyama et al, Phys. Rev. Lett. 65, 3156 (1990); A. Koyama, Nuc. Inst. Meth. 67, 103 (1992);

[8] H. Ishikawa et al, Nuc. Inst. Meth. 67, 160 (1992).

[9] M. Hasegawa, T. Fukuchi, Y. Mizuno, K. Kimura, and M. Mannami, Nuc. Inst. Meth. B53, 285 (1991).

[10] K. Kimura, M. Tsuji, and M. Mannami, Phys. Rev. A 46, 2618 (1992).

[11] E. A. Sánchez, O. Grizzi, M. L. Martiarena, and V. H. Ponce, Phys. Rev. Lett. 71, 801 (1993).

[12] H. Lebius, R. Minniti, J.Y. Lim, and S. Elston, to be published in Phys Rev A.

[13] R. A. Baragiola, Nuc. Inst. Meth. B78, 223 (1993).

[14] T. Iitaka, Y. H. Ohtsuki, A. Koyama, and H. Ishikawa, Phys. Rev. Lett. 65, 3160 (1990).

[15] D.R. Schultz, C.O. Reinhold, and R.E. Olson, in Two Center Effects in Ion-Atom Collisions, Edited by T.j. Gay and A.F. Starace (AIP Conf. Proc. 362, 1996), p. 84; C. O. Reinhold and R. E. Olson, Phys. Rev. A 39, 3861 (1989); R. L. Becker and A. D. MacKellar, J. Phys. B 17, 3923 (1984); C. O. Reinhold and C. A. Falcón, Phys. Rev. A 33, 3859 (1986).

[16] R. Abrines and I. C. Percival, Proc. Phys. Soc. London 88, 861 (1966).

[17] J. Burgdörfer and J. Gibbons, Phys. Rev. A 42 (1990) 1206; J. Burgdörfer, Lecture Notes in Physics 294 (1988) 344; J. Burgdörfer, Lecture Notes in Physics 376 (1990) 199. C. O. Reinhold, J. Burgdörfer, J. Kemmler, and P. Koschar, Phys. Rev. A 45 (1992) R2655.

[18] C. O. Reinhold, J. Burgdörfer, K. Kimura, and M Mannami Phys. Rev. Lett. 73, 2508 (1994).

[19] C. O. Reinhold and J. Burgdörfer, submitted to Phys Rev A (1996).

[20] J. Burgdörfer, Review of Fundamental Processes and Applications of Atoms and Ions, ed. C.D. Lin (World Scientific, Singapore 1993). 
[21] R. Minniti, J.Y. Lim, and S. Elston, in preparation.

[22] K. Kimura, T. Kishi, and M. Mannami, Nucl. Inst. Meth. B 90 (1994) 282.

[23] K. Kimura, M. Hasegawa, and M. Mannami, Phys. Rev. A 36, 7 (1987).

[24] F. J. García de Abajo and P.M. Echenique, Phys. Rev. B 46, 2663 (1992).

[25] P. M. Echenique, F. Flores, and R. H. Ritchie, Solid State Phys. 43, 229 (1990).

[26] T. F. M. Bonsen and L. Vriens, Physica 47, 307 (1970); M.E. Rudd, C.A. Sauer, and C.L. Bailey, Phys. Rev. 151, 20 (1966); Lee et al, Phys. Rev. A 41, 4816 (1990).

[27] M. R. C. McDowell and J. P. Coleman Introduction to the Theory of Ion-Atom Collisions (North-Holland, Amsterdam, 1970).

[28] R. Pfandzelter, private communication (1995); T. Berner, Diploma Thesis, Humboldt Universität; A. Hegmann et al, Europhys. Lett. 26, 383 (1994).

[29] K. Kimura, private communication (1995).

[30] J. M. Pitarke, A. Bergara, and R.H. Ritchie, Nuc. Instr. Meth. B99, 187 (1995). 


\section{Figure Captions}

Figure 1: Position of ion image charge at large distances d.

Figure 2: The ionic trajectory, $R_{z}\left(R_{x}\right)$, the typical stochastic charge state evolution, $Q_{p}\left(R_{x}\right)$, and the charge state fractions for an incident $0.3 \mathrm{MeV} / \mathrm{u} \mathrm{Li}{ }^{+}$projectile on $\mathrm{SnTe}$ surfaces. The solid squares are experimental data of Kimura et al [22] for outgoing charge state fractions. The solid is contained in the $z<0$ region and the surface is contained in the $(\mathrm{x}, \mathrm{y})$ plane.

Figure 3: Experimental outgoing charge state fractions for grazing incidence $0.5 \mathrm{MeV} / \mathrm{u}$ $C^{q+}$ ions impinging on $\mathrm{Si}$ surfaces.

Figure 4: Potential for an electron initially in a target core (T) near a $0.3 \mathrm{MeV} / \mathrm{u} \mathrm{Li}^{2+}$ ion (P) moving parallel to a $\mathrm{SnTe}(001)$ surface as a function of the position vector of the electron $(x, y=1, z)$. The position of the projectile is $(0,0,-1)$ and the target core is at $(20,0,-3)$.

Figure 5: Density plot in velocity space of ejected valence or core electrons arising from $0.3 \mathrm{MeV} / \mathrm{u}$ Li-SnTe collisions cut in the $\left(v_{x}, v_{z}\right)$ plane. The thick line indicates the location of the binary ridge. The void in the center is due to the fact that we consider only fast electrons with $v_{e}>v_{p} / \sqrt{2}$.

Figure 6: Effect of the wake potential $V_{p e}^{I}$ on the spectrum of ejected electrons. As in Fig. 11, however sum of the core and valence electrons.

Figure 7: Doubly differential yield of electrons per impinging ion in $0.3 \mathrm{MeV} / \mathrm{u} \mathrm{Li}$ SnTe collisions as a function of the electron energy for different emission angles with respect to the $\mathrm{x}$-axis: total yield (thick solid line), valence electrons (thin solid line), core electrons (dashed line), electrons which were transiently captured to bound states of the ions (dashed dotted line).

Figure 8: Triply differential yield of electrons emitted at $\theta=98 \mathrm{mrad}$ in the $(\mathrm{x}, \mathrm{z})$ plane in a solid angle of $100 \times 100 \mathrm{mrad}^{2}$ resulting from the interaction of $0.2 \mathrm{MeV} / \mathrm{u} \mathrm{Li}$ ions with $\mathrm{SnTe}(001)$ : calculated total yield (solid lines), calculated yield of valence electrons (dashed lines) and experiment (open circles). The experimental data [22] have been normalized to theory. The calculated and experimentally estimated yields per ion within the FWHM are given by $Y_{t h}$ and $Y_{e x p}$, respectively. 


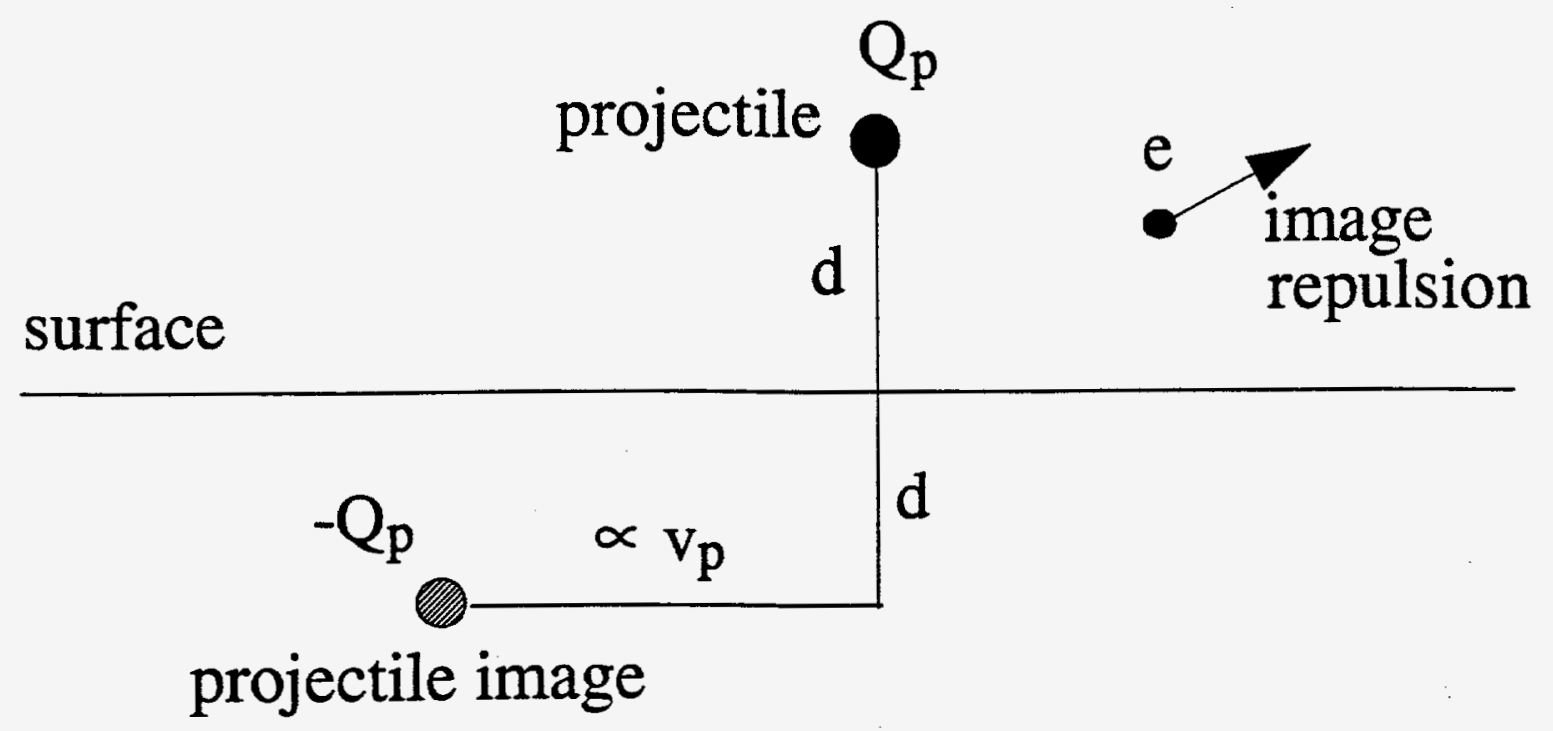

Figure 1 


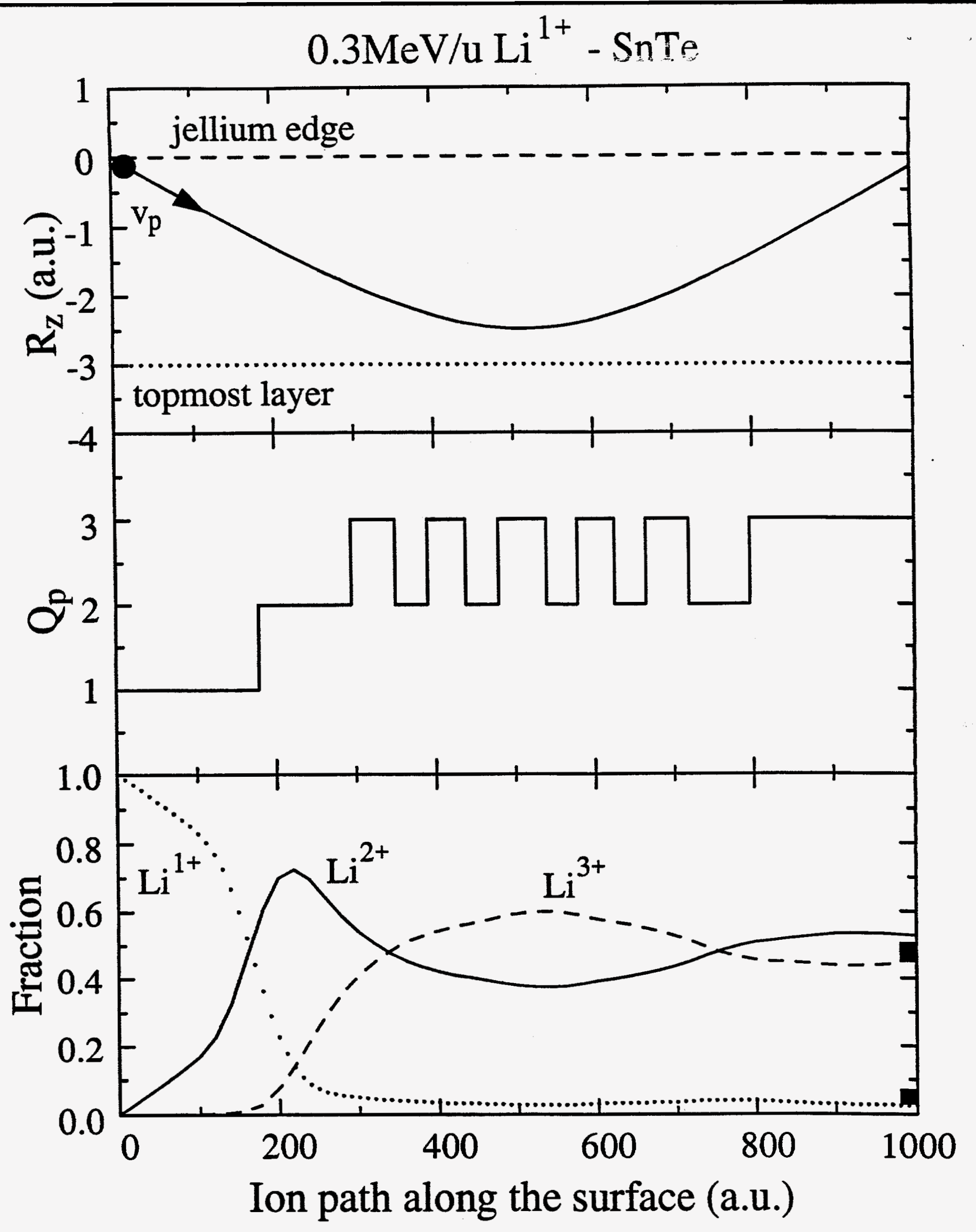

Figure 2 
$0.5 \mathrm{MeV} / \mathrm{u} \mathrm{Cq}+-\mathrm{Si}$

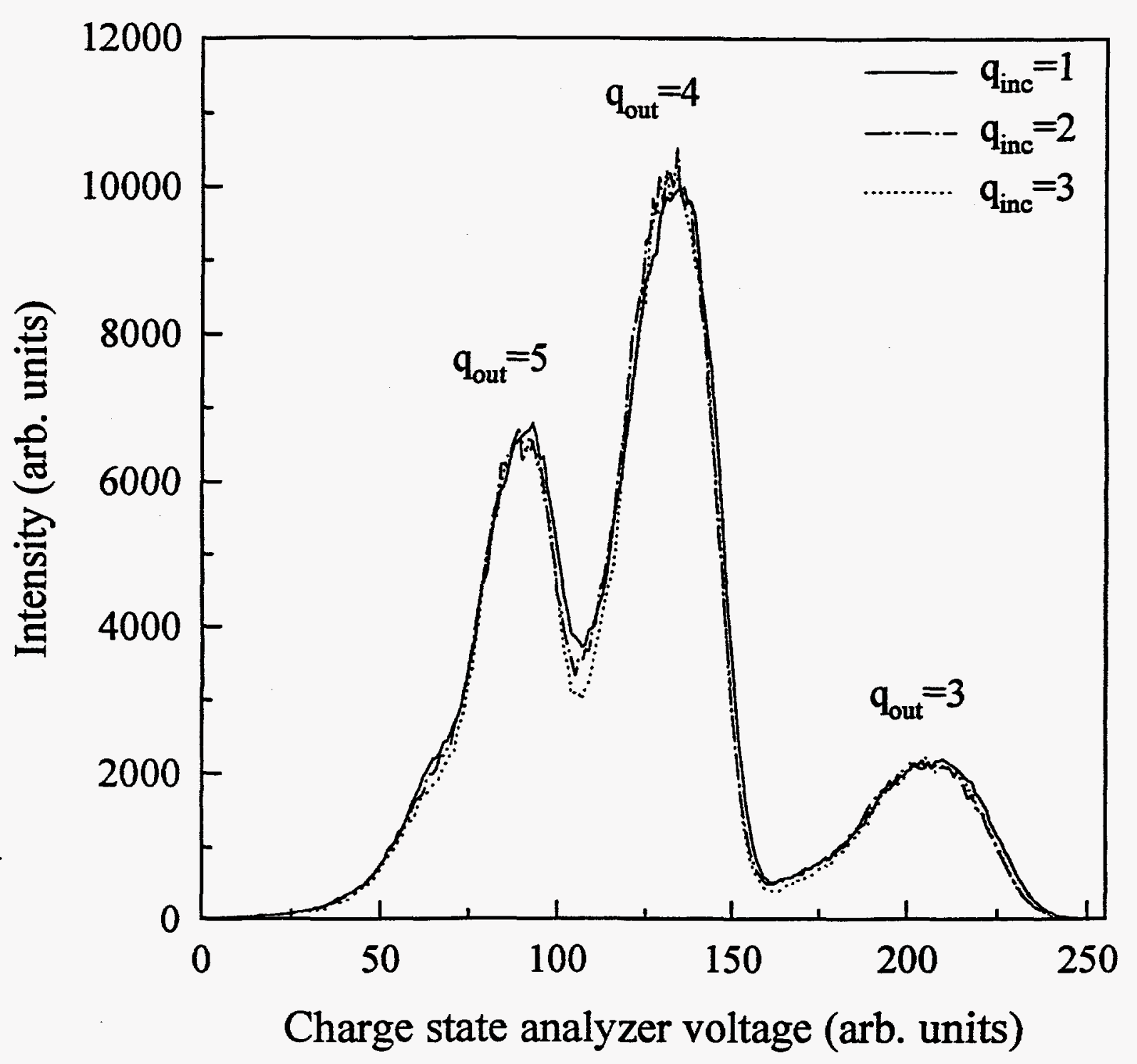

Figure 3 


\section{$0.3 \mathrm{MeV} / \mathrm{u} \mathrm{Li}^{2+}-\mathrm{SnTe}$}

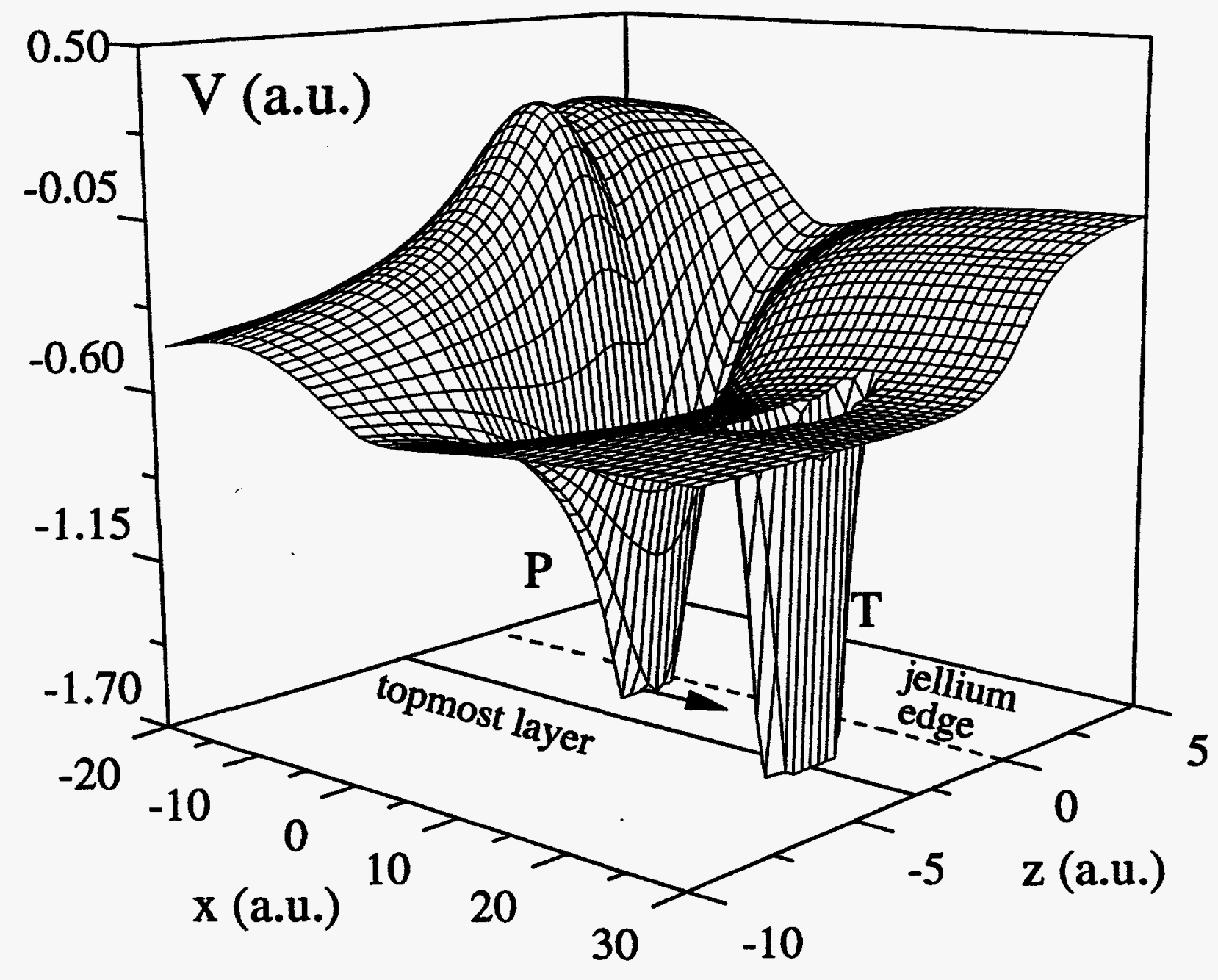

Figure 4 


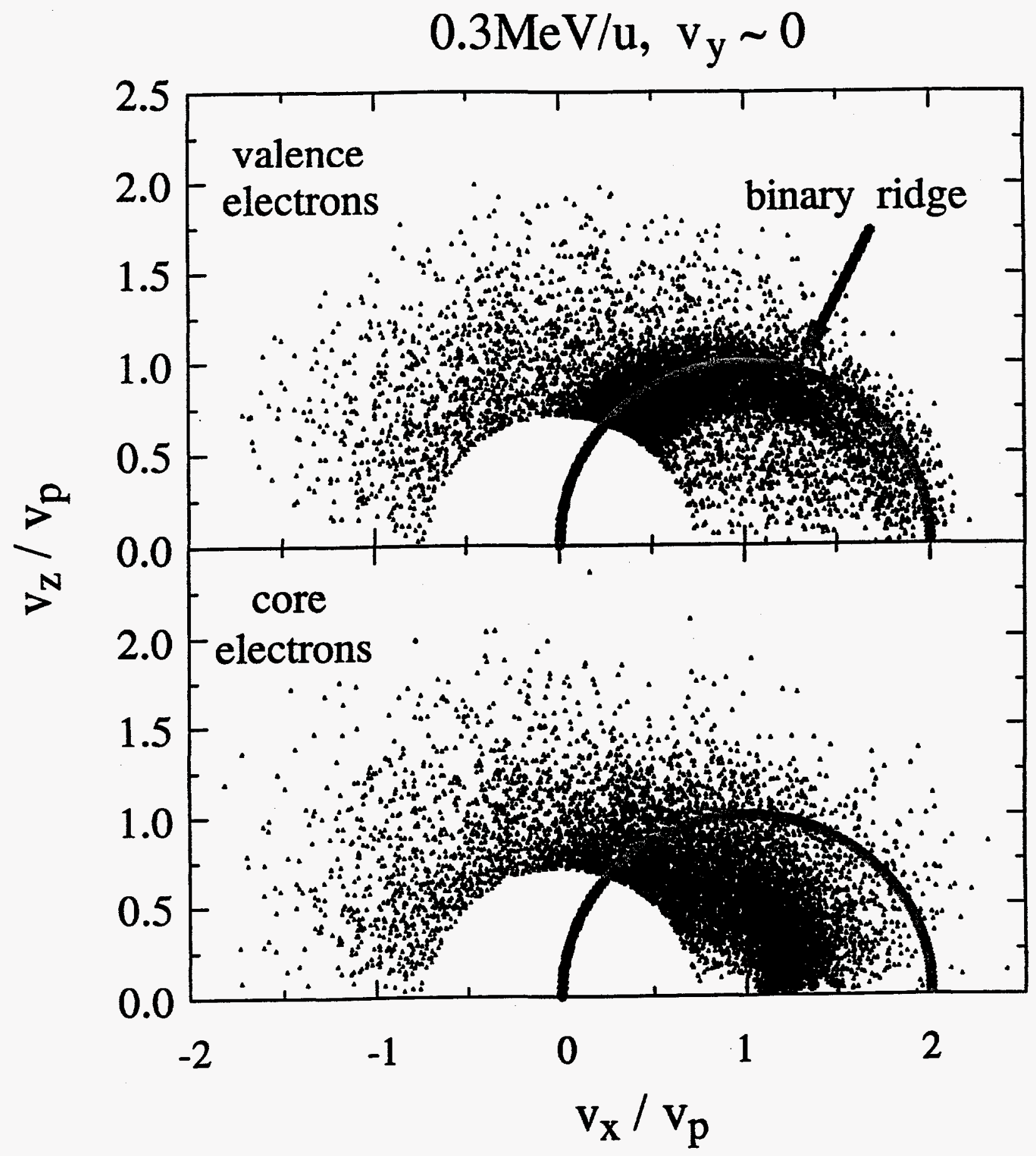

Figure 5 


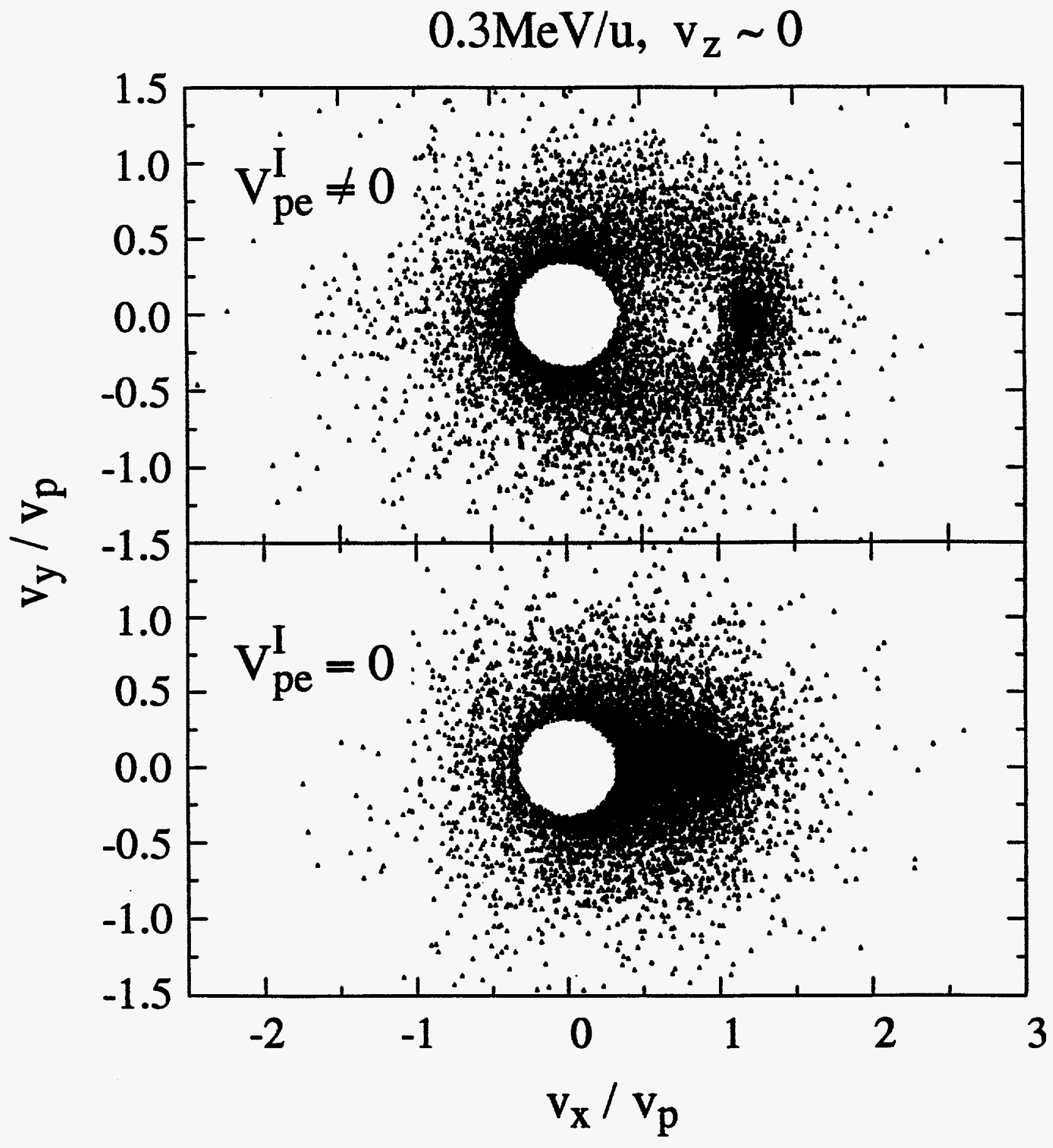

Figure 6 


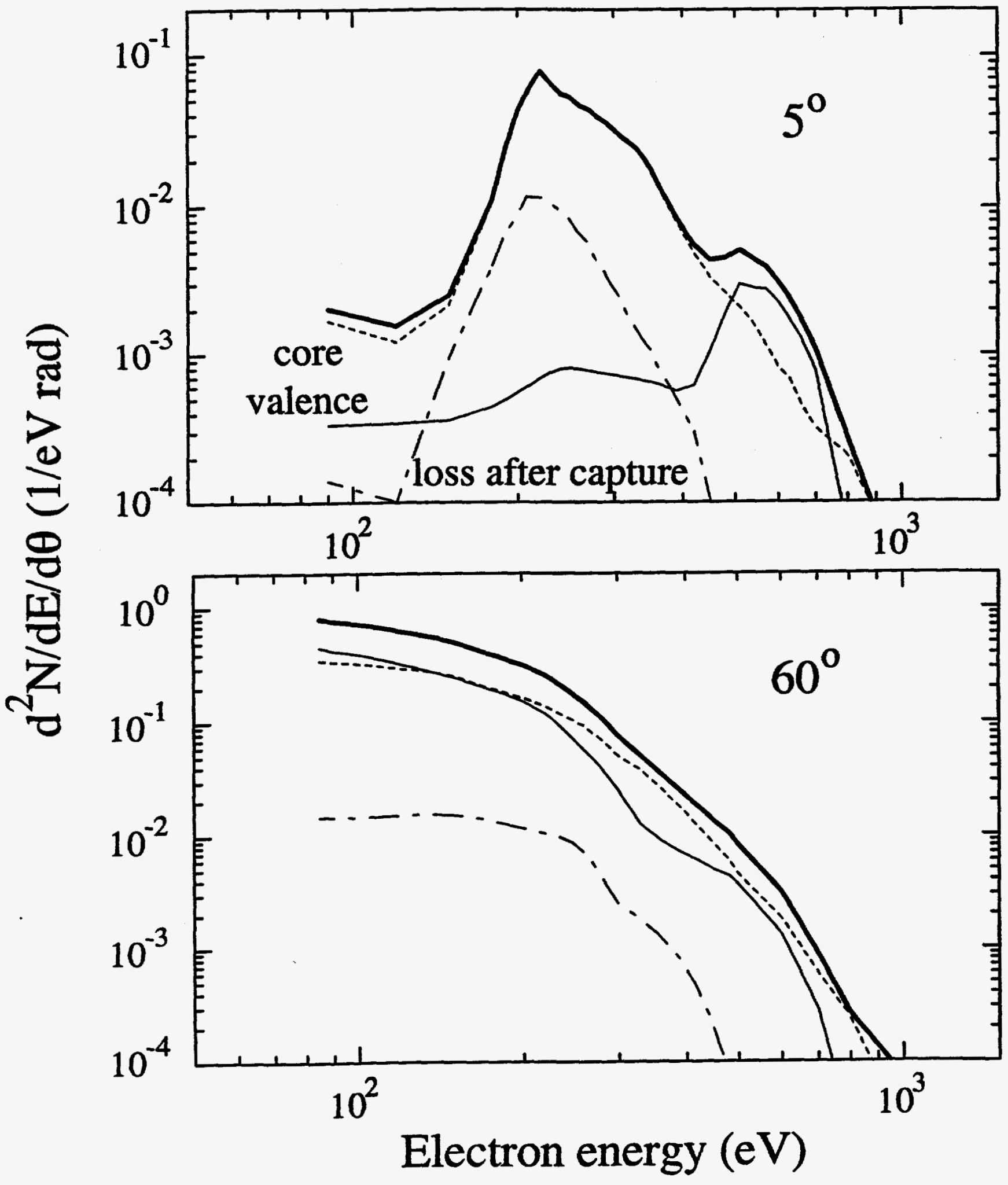

Figure 7 


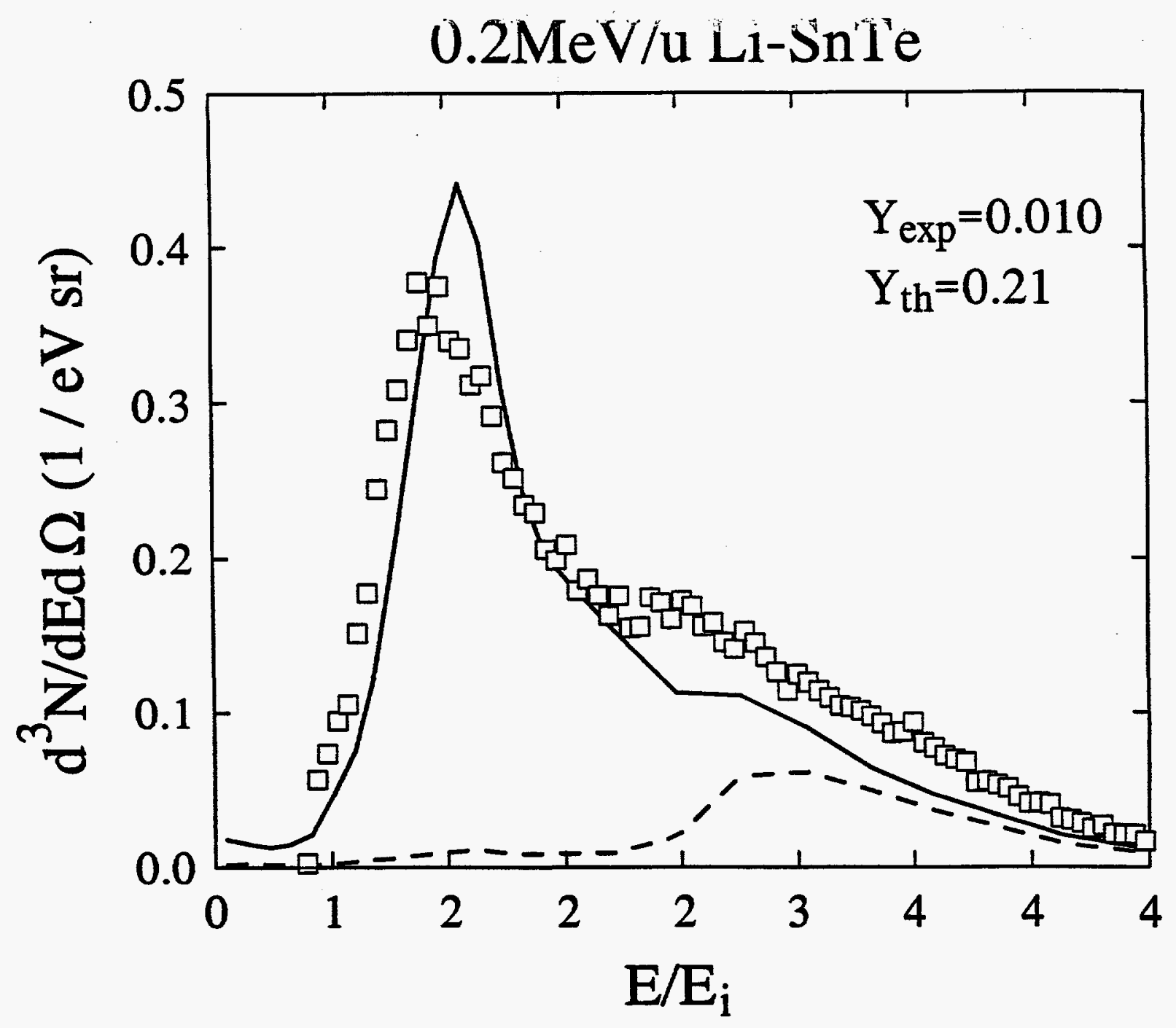

Figure 8 